\title{
Connectomics and Neuroticism: An Altered Functional Network Organization
}

\author{
Michelle N Servaas*,', Linda Geerligs',2, Remco J Renken', Jan-Bernard C Marsman', Johan Ormel', \\ Harriëtte Riese ${ }^{3}$ and André Aleman ${ }^{1,2}$ \\ 'Department of Neuroscience, Neuroimaging Center, University Medical Center Groningen, University of Groningen, Groningen, The Netherlands; \\ ${ }^{2}$ Department of Psychology, University of Groningen, Groningen, The Netherlands; ${ }^{3}$ Department of Psychiatry, Interdisciplinary Center for \\ Psychopathology and Emotion Regulation, University Medical Center Groningen, University of Groningen, Groningen, The Netherlands
}

\begin{abstract}
The personality trait neuroticism is a potent risk marker for psychopathology. Although the neurobiological basis remains unclear, studies have suggested that alterations in connectivity may underlie it. Therefore, the aim of the current study was to shed more light on the functional network organization in neuroticism. To this end, we applied graph theory on resting-state functional magnetic resonance imaging ( $\mathrm{MRR}$ ) data in 120 women selected based on their neuroticism score. Binary and weighted brain-wide graphs were constructed to examine changes in the functional network structure and functional connectivity strength. Furthermore, graphs were partitioned into modules to specifically investigate connectivity within and between functional subnetworks related to emotion processing and cognitive control. Subsequently, complex network measures (ie, efficiency and modularity) were calculated on the brain-wide graphs and modules, and correlated with neuroticism scores. Compared with low neurotic individuals, high neurotic individuals exhibited a whole-brain network structure resembling more that of a random network and had overall weaker functional connections. Furthermore, in these high neurotic individuals, functional subnetworks could be delineated less clearly and the majority of these subnetworks showed lower efficiency, while the affective subnetwork showed higher efficiency. In addition, the cingulo-operculum subnetwork demonstrated more ties with other functional subnetworks in association with neuroticism. In conclusion, the 'neurotic brain' has a less than optimal functional network organization and shows signs of functional disconnectivity. Moreover, in high compared with low neurotic individuals, emotion and salience subnetworks have a more prominent role in the information exchange, while sensory(-motor) and cognitive control subnetworks have a less prominent role.

Neuropsychopharmacology (2015) 40, 296-304; doi: I0.1038/npp.20I4.169; published online 6 August 20I4
\end{abstract}

\section{INTRODUCTION}

Neuroticism is a fundamental part of various widely accepted personality taxonomies (Costa and McCrae, 1992; Eysenck, 1967; Gray, 1991) and is a highly efficient marker of risk for psychopathology, specifically depression and anxiety disorders (Lahey, 2009; Ormel et al, 2013b). High compared with low neurotic individuals tend to interpret events as more threatening ('negativity bias'), react more emotionally to negative events and apply maladaptive coping strategies in the face of stressors (Suls and Martin, 2005; Watson et al, 1994).

Early electrophysiological studies (Eysenck, 1967; Gray, 1991) as well as recent neuroimaging studies (Ormel et al, 2013a) on the biological basis of neuroticism have inve-

*Correspondence: Dr MN Servaas, Department of Neuroscience, Neuroimaging Center, University Medical Center Groningen, University of Groningen, PO Box 196, 9700 AD, Groningen, The Netherlands, Tel: +3I 050363 8792, Fax: +31 0503638875 ,

E-mail: m.n.servaas@umcg.nl or m.n.servaas@gmail.com

Received 28 April 2014; revised 25 June 2014; accepted I July 20।4; accepted article preview online 9 July 2014 stigated emotional reactivity and emotion regulation, and proposed a dysfunctional system of limbic and frontal brain regions to underlie abovementioned difficulties in high neurotic individuals. Indeed, recent structural and functional connectivity studies have demonstrated alterations in limbic-frontal circuitry in association with neuroticism, possibly compromising top-down control processes (Bjørnebekk et al, 2013; Cremers et al, 2010; Servaas et al, 2013a; Xu and Potenza, 2012). Notably, structural connectivity studies showed decreased white-matter integrity not only in fiber tracts interconnecting PFC regions and the amygdala, but in multiple other fiber tracts as well (Bjørnebekk et al, 2013; Xu and Potenza, 2012). Due to the wide distribution of the observed effects, these studies suggested that general disconnectivity may potentially underlie the predisposition to experience negative affect (Bjørnebekk et al, 2013; Xu and Potenza, 2012).

To investigate whether there is also evidence for a disruption of whole-brain functional connectivity in association with neuroticism, graph theory analysis (GTA) was performed on resting-state functional magnetic resonance imaging (rs-fMRI) data in the current study. This analysis method explores the topological properties of complex networks by calculating measures that provide information 
on functional integration and segregation. A commonly used measure of functional integration is efficiency, which measures the capability of the network to process information rapidly in a parallel manner. A refined measure of functional segregation is modularity, which quantifies the degree to which a network can be clearly delineated in non-overlapping groups of nodes (brain regions) (Newman, 2004; Rubinov and Sporns, 2010). These network measures can be calculated on binary networks-wherein an edge (connection) is indicated as present or not-providing information on the functional network structure or on weighted networks-wherein edge strengths are preservedadditionally providing information on the functional connectivity strength (Rubinov and Sporns, 2010). Studies using the graph theory method have repeatedly shown that complex networks, such as the brain, exhibit a small-world organization, that is, nodes are highly clustered in specialized local networks (resulting in a higher local efficiency than in random networks), while, simultaneously, information between any pair of them can be transported via a minimal number of edges (resulting in a higher global efficiency than in regular networks) (Watts and Strogatz, 1998; Xia and He, 2011).

Besides characterizing the topological properties of the whole-brain network, it is important to describe the properties of the different functional subnetworks to understand how the whole-brain network is organized and the way information is integrated within and between functional subnetworks. It would be of specific interest to investigate the role that subnetworks related to emotion processing and cognitive control play in the network organization of individuals scoring higher on neuroticism, since these individuals show difficulties in adaptive emotion regulation (Suls and Martin, 2005; Watson and Hubbard, 1996; Yoon et al, 2013). In the study of Geerligs et al (2014), the network measure local efficiency was used to define functional integration within a network and the participation coefficient was used to define functional integration between networks (Geerligs et al, 2014). The latter coefficient measures the degree to which a node is connected to other nodes that are part of a different functional subnetwork (Rubinov and Sporns, 2010). To our knowledge, only one graph theoretical study (based on the whole-brain network) has been conducted on neuroticism so far (Gao et al, 2013). An important finding of this study was that the amygdala functions more as a hub in high neurotic individuals compared with low neurotic individuals.

The aim of the current study was to investigate alterations in the functional network organization associated with neuroticism. We applied GTA on rs-fMRI data in a sample of 120 women selected on the basis of their neuroticism score. We hypothesized to observe a less than optimal functional organization (Jin et al, 2011; Zhang et al, 2011) and signs of functional disconnectivity (Bjørnebekk et al, 2013; Xu and Potenza, 2012) in the network of individuals with higher levels of neuroticism, that is, altered efficiency and less clearly delineated functional subnetworks. Furthermore, we hypothesized that subnetworks related to emotion processing have a more prominent role in the network organization of these individuals than subnetworks related to cognitive control (Ormel et al, 2013a).

\section{MATERIALS AND METHODS}

\section{Participants}

Initially, 240 students from the University of Groningen were asked to fill out the NEO Five-Factor Inventory (NEOFFI) (domains Neuroticism and Extraversion, 24 items). Individuals were sent a questionnaire when they agreed to participate in the study (based on the information letter, which included an informed consent form) and met the following selection criteria: (1) female gender, (2) age between 18 and 25 years, (3) Dutch as native language, (4) Caucasian descent, (5) right handed, and (6) no use of contraceptive medication, except for oral contraceptive pills (21-pill packet). Only females were included because they significantly score higher on neuroticism than men, and because of that have a higher risk of developing affective disorders (Parker and Brotchie, 2010). Furthermore, research is still limited related to gender differences in neuroticism and therefore, we decided not to introduce this variation in the sample as it is not properly understood yet. Exclusion criteria were (1) a history of seizure or head injury, (2) a lifetime diagnosis of psychiatric and/or neurological disorders, (3) a lifetime diagnosis of psychiatric disorders in first-degree relatives of the participant, (4) the use of medication that can influence test results, (5) visual or auditory problems that cannot be corrected, (6) MRI incompatible implants or tattoos, (7) claustrophobia, and (8) suspected or confirmed pregnancy. From this sample, 120 individuals (mean age: $20.8 \mathrm{SD} \pm 2.0$, age range: $18-25$ ) were invited to participate in the experiment. To ensure sufficient numbers of participants with high levels of neuroticism, 60 individuals were selected from the highest quartile of neuroticism scores (NEO-FFI score $\geqslant 32$, range 32-47) and 60 individuals were randomly selected from the three lowest quartiles (NEO-FFI $<32$, range 17-31). Plots of normality (QQ-plot and boxplot) showed that, in the selected 120 participants, neuroticism scores were approximately normally distributed.

To reduce hormone-related between-subject variability, participants were invited for the experiment during the first 10 days of their menstrual cycle (early and mid-follicular phase) or during the discontinuation week in case of oral contraceptive usage, which resembles the early and midfollicular phase in terms of ovarian hormonal levels (Cohen and Katz, 1979). During these phases, ovarian hormonal levels are relatively low and menstrual cycle-related changes in mood, stress sensitivity, and neurocognitive function are minimal (Andreano and Cahill, 2010; Goldstein et al, 2010; Symonds et al, 2004).

On the day of the experiment, after explaining the procedure, participants gave informed consent again and completed the NEO Personality Inventory Revised (NEO-PI-R) (domains Neuroticism, Extraversion, and Conscientiousness, 144 items) (Costa and McCrae, 1992). The mean neuroticism score across the whole sample was $135.5 \pm$ SD 19.0 (range: 94-195). The study was approved by the Medical Ethical Committee of the University Medical Center Groningen and was conducted in accordance with the Declaration of Helsinki.

\section{Image Acquisition}

A 3 Tesla Phillips Intera MRI scanner (Phillips Medical Systems, Best, The Netherlands), equipped with a 32-channel SENSE 
head coil, was used to acquire the images. A high-resolution T1-weighted 3D structural image was obtained using fast-field echo (FFE) for anatomical reference (170 slices; TR: $9 \mathrm{~ms}$; TE: $8 \mathrm{~ms}$; FOV: $256 \times 231 ; 256 \times 256$ matrix; voxel size: $1 \times 1 \times$ $1 \mathrm{~mm}$ ). rs-fMRI images were acquired with $\mathrm{T} 2{ }^{*}$-weighted gradient echo planar imaging (EPI) sequences. Participants were instructed to close their eyes and to not fall asleep. The scan comprised 300 volumes of 37 axial-slices (TR: $2000 \mathrm{~ms}$; TE: $30 \mathrm{~ms}$; FOV: $220 \times 221$; $64 \times 62$ matrix; voxel size: $3.5 \times$ $3.5 \times 3.5 \mathrm{~mm}$ ). Slices were acquired in descending order without a gap. To prevent artifacts due to nasal cavities, images were tilted $10^{\circ}$ to the AC-PC transverse plane (see Supplementary Material S1 for more details on the full fMRI session).

\section{Data Preprocessing}

Image processing was performed using SPM8 (http:// www.fil.ion.ucl.ac.uk/spm), implemented in Matlab 7.8.0 (The Mathworks Inc., Natick, MA). Preprocessing included realignment, coregistration, DARTEL normalization $(2 \times$ $2 \times 2 \mathrm{~mm}$ isotropic voxels) (Ashburner, 2007) and smoothing $(8 \mathrm{~mm}$ full-width at half maximum (FWHM) Gaussian kernel) (see Supplementary Material S2 for details on the preprocessing steps).

Next, a series of preprocessing steps specific to rs-fMRI analysis were performed. First, regression of several nuisance variables was applied per gray-matter voxel to remove sources of spurious variance, comprising six rigid body head motion parameters, the global signal, whitematter (WM) signal and cerebrospinal fluid (CSF) signal. To obtain the last two signals, we performed segmentation to create a WM and CSF mask and extracted the first eigenvariate from the time series of the included voxels. In addition, the first temporal derivatives of abovementioned nuisance variables were removed. Second, temporal band-pass filtering was applied to detrend the signal and to retain frequencies between 0.008 and $0.08 \mathrm{~Hz}$ (Van Dijk et al, 2010). Third, we performed scrubbing to additionally remove influences of movement on the rs-fMRI data (Power et al, 2012) (see Supplementary Material S3 for details on the scrubbing procedure).

Finally, eight subjects were excluded from further analysis; two because of anatomical abnormalities, four because of technical difficulties, and two because of scrubbing. A total sample of 112 subjects remained for statistical analysis.

\section{Network Construction}

To perform GTA, nodes and edges have to be defined. For nodes, a sphere of 5- $\mathrm{mm}$ radius was created around 264 coordinates provided by Power et al (2011). After visual inspection of the regions of interest (ROIs), we noted the absence of three relevant subcortical structures for research on neuroticism: bilateral amygdala, hippocampus, and caudate (Servaas et al, 2013b). The coordinates for these regions were determined using the Harvard-Oxford Subcortical Structural Atlas ( $80 \%$ probability), resulting in a total of 270 ROIs. No overlap was observed between the additional ROIs and the ROIs of Power et al (2011). Next, we binarized the functional images of all subjects and built a whole-brain group mask by multiplying them. This locates the parts of the brain, where EPI images were free from susceptibility artifacts in all subjects. Subsequently, the overlap was calculated voxelwise between all ROIs and the group mask. When a ROI overlapped $<50 \%$ with the group mask, it was excluded from further analysis. This was the case for 11 ROIs. To construct a connectivity matrix per subject, we extracted the regional mean time series for each of the remaining 259 ROIs and calculated Pearson correlations between all pairs. Furthermore, to prevent biases due to shared nonbiological signals between adjacent ROIs, correlations were set to zero when the distance was $<20 \mathrm{~mm}$ between the centers of two ROIs (Power et al, 2011). In addition, correlations on the diagonal of the connectivity matrix were set to zero as well.

\section{Thresholding}

It has been shown that the majority of network measures are highly sensitive to the number of edges in a graph (van Wijk et al, 2010). To avoid this confound, we applied a range of proportional thresholds to each correlation matrix per subject. The threshold values $(T)$ ranged from 0.01 to 0.30 , in increments of 0.01 . Network measures were calculated on the whole-brain and different functional subnetworks for both binary and weighted graphs across the selected range of threshold values (see below). To partition the graph into modules, we applied the algorithm of Blondel et al, 2008 and the modularity fine-tuning algorithm of Sun et al, 2009. For this procedure, we selected a single optimal threshold by applying the method described in Geerligs et al (2014). The optimal threshold in the current study was $1.8 \%$ (see Supplementary Material S4 for details on the selection of the optimal threshold and see Supplementary Material S5 for details on the module decomposition).

\section{Network Measures}

Network measures were calculated on binary and weighted graphs across the selected range of threshold values by using functions implemented in the Brain Connectivity Toolbox (www.brain-connectivity-toolbox.net, Rubinov and Sporns, 2010). First, we calculated the whole-brain network measures: global efficiency $\left(E_{\text {global }}\right)$, local efficiency $\left(E_{\text {local }}\right.$, averaged across nodes) and maximized modularity $(Q)$. Global efficiency is calculated as the average inverse shortest path length between all pairs of nodes. Local efficiency is calculated in a similar manner but then between a node and its direct neighbors (Latora and Marchiori, 2001; Rubinov and Sporns, 2010). Maximized modularity is calculated with a function that quantifies the degree to which a network can be clearly delineated in non-overlapping groups of nodes (Newman, 2004; Rubinov and Sporns, 2010). Second, we calculated local efficiency ( $E_{\text {local }}$, averaged across nodes) and the participation coefficient ( $Y$, averaged across nodes) per module (see Supplementary Material S5 for details on the module decomposition). The participation coefficient is calculated as the ratio of intra- $v s$ intermodular connections per node (Rubinov and Sporns, 2010). Third, Pearson correlations were calculated between neuroticism and abovementioned network measures across the selected range of threshold values (see Supplementary Material S6 Figure 1 for exemplars showing the linear 
association between neuroticism and the network measures). The correlation values were plotted (see Figures 1 and 2) and visually checked for consistency across threshold values. Subsequently, we calculated the area under the curve (AUC) across threshold values per network measure to obtain a summarized scalar that is independent of single threshold selection. Next, non-parametric permutation testing was applied on the AUC per network measure to assess whether the results could have occurred by chance. To this end, neuroticism scores were permuted randomly and correlations with each network measure were recalculated. This procedure was repeated 5000 times and a one-tailed test of the null hypothesis $(P<0.05)$ was performed per network measure (for details of this method, see Zhang et al, 2011).
Fourth, we investigated the association between neuroticism and the overall functional connectivity strength. The reason for this was that we found different results for global efficiency between binary and weighed graphs in relation to neuroticism (see Results, Whole-Brain Network Measures). As a measure for the overall functional connectivity strength, we calculated the median of the positive, pairwise correlation values between ROIs per subject. Positive correlation values were selected, because we applied a proportional threshold (ie, $\mathrm{k} \%$ strongest connections) when calculating the network measures. Consequently, the median was chosen over the mean, because distributions were highly asymmetric. Next, a Spearman rank correlation was calculated between neuroticism and these median values.
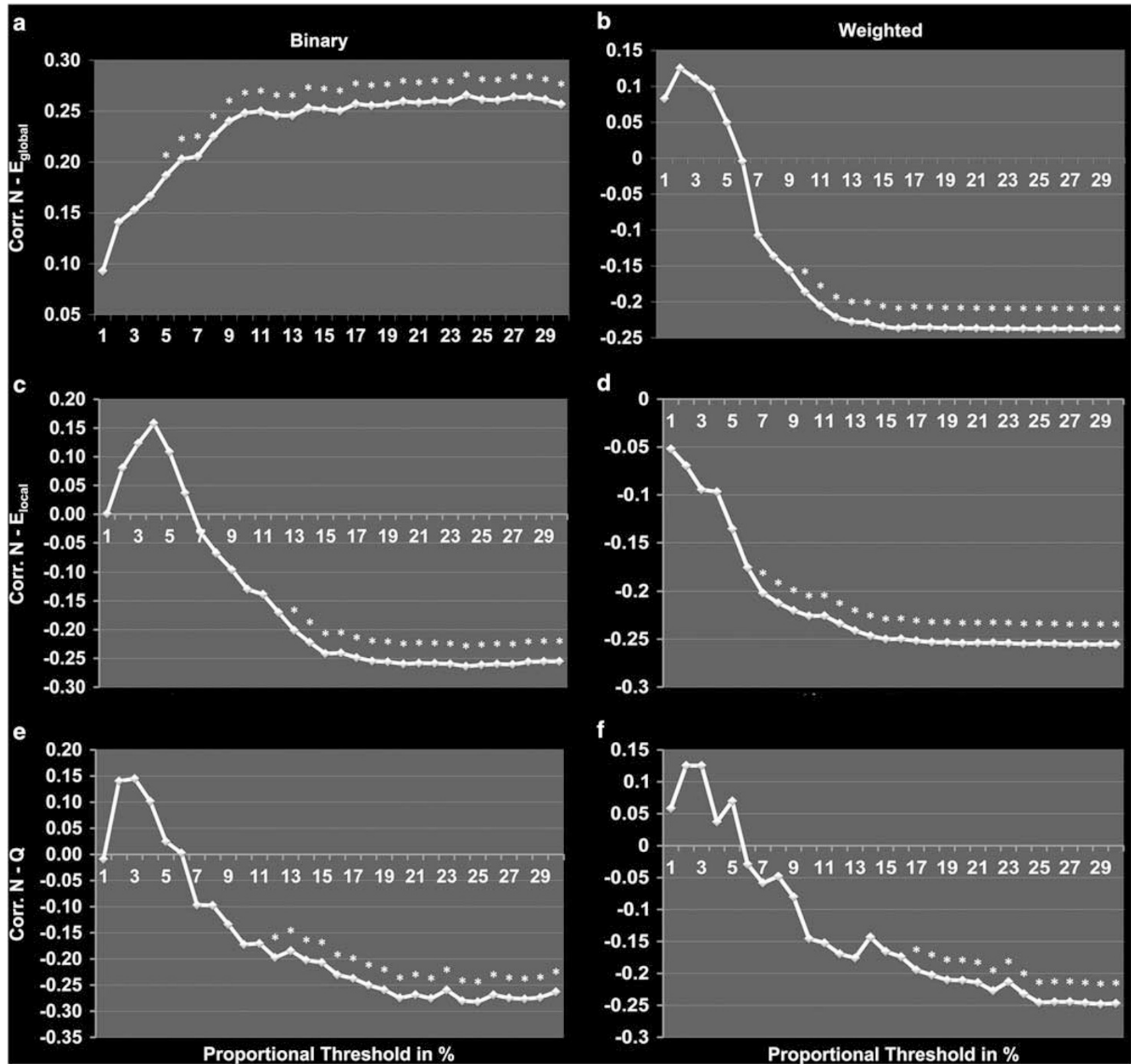

Proportional Threshold in \%

Proportional Threshold in \%

Figure I Correlations between neuroticism and the whole-brain network measures. In the panels, correlations between neuroticism, and global efficiency $\left(E_{\text {global }}\right)(a, b)$, local efficiency $\left(E_{\text {local }}\right)(c, d)$, and maximized modularity $(Q)(e, f)$ can be observed over a range of threshold values $(T=0.0 \mathrm{I}-0.30$, in increments of $0.0 \mathrm{I})$ for binary ( $a, c$, and e) and weighted graphs (b, d, and f). Significant correlations $(p<0.05$, uncorrected for the number of threshold values) are marked with an asterisk (*). 


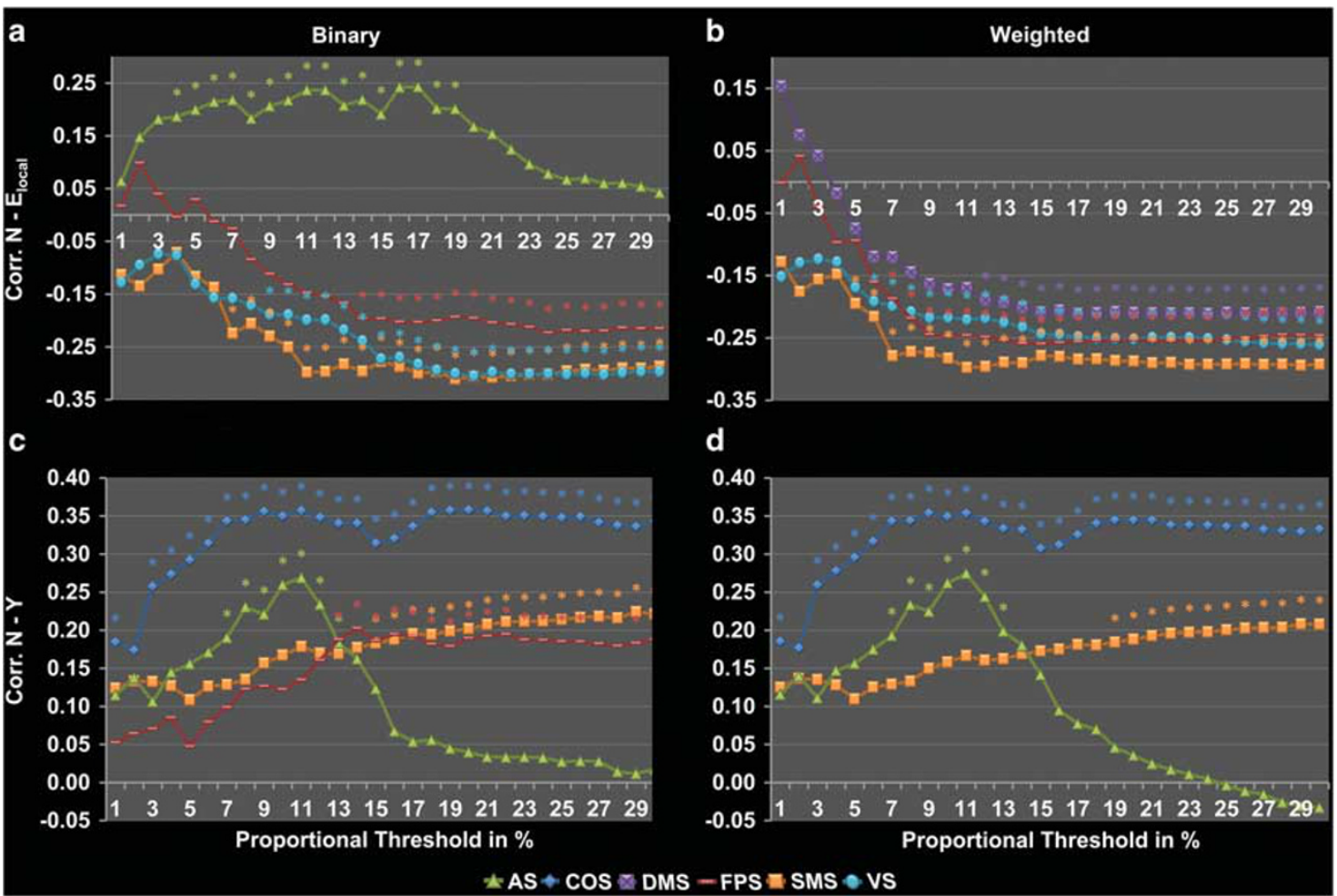

Figure 2 Correlations between neuroticism and the network measures per module. In the panels, correlations between neuroticism, and local efficiency $\left(E_{\text {local }}\right)(a, b)$ and the participation coefficient $(Y)(c, d)$ can be observed over a range of threshold values $(T=0.0 \mathrm{I}-0.30$, in increments of $0.0 \mathrm{I})$ for binary $(\mathrm{a}, \mathrm{c})$ and weighted graphs (b, d) per module. Colors indicate the different modules: AS, affective subnetwork (green); COS, cingulo-operculum subnetwork (dark blue); DMS, default mode subnetwork (purple); FPS, fronto-parietal subnetwork (red); SMS, somatosensory-motor subnetwork (orange); and VS, visual subnetwork (light blue). Significant correlations $(p<0.05$, uncorrected for the number of threshold values) are marked with an asterisk $(*)$. Only subnetworks, for which significant results were found based on permutation testing, are shown.

\section{RESULTS}

\section{Whole-Brain Network Measures}

For binary graphs, we found a positive correlation between neuroticism and global efficiency. For weighted graphs, a negative correlation was observed between neuroticism and global efficiency. Furthermore, for both types of graphs, neuroticism was negatively correlated with local efficiency and maximized modularity (see Figure 1 and Table 1 for the results).

Next, a Spearman rank correlation was calculated between neuroticism and the overall functional connectivity strength to investigate the different results found for global efficiency between binary and weighted graphs. A significant negative correlation was found $(r=-0.23, p=0.016)$, which indicates that individuals scoring higher on neuroticism have overall weaker functional connections (see Supplementary Material S7 Figure 2 showing the association between neuroticism and the overall functional connectivity strength).

\section{Network Measures Per Module}

Nodes could be partitioned in six functional subnetworks with a maximum number of within-group edges and a minimum number of between-group edges (Blondel et al, 2008; Rubinov and Sporns, 2010; Sun et al, 2009). These included the affective subnetwork (AS), cingulo-operculum subnetwork (COS), default mode subnetwork (DMS), fronto-parietal subnetwork (FPS), somatosensory-motor subnetwork (SMS), and visual subnetwork (VS) (see Figures 3 and 4). The modular structure was comparable to the one found by Power et al (2011) and other robust functional subnetwork decompositions observed in the resting-state literature (eg, Damoiseaux et al, 2006).

For binary graphs, we found a negative correlation between neuroticism and local efficiency in the FPS, SMS, and VS. In contrast, neuroticism was positively associated with local efficiency in the AS. For weighted graphs, we also observed a negative correlation between neuroticism and local efficiency in the FPS, SMS, and VS. However, no positive association was observed between neuroticism and local efficiency in the AS. Additionally, a negative correlation was found between neuroticism and local efficiency in the DMS for weighted graphs (see Figure 2 and Table 1 for the results).

For the participation coefficient calculated on binary graphs, a positive correlation was identified with neuroticism in the AS, COS, FPS, and SMS. For weighted graphs, neuroticism also showed a positive correlation with this coefficient in the AS, COS, and SMS, but not in the FPS (see Figure 2 and Table 1 for the results).

\section{DISCUSSION}

The aim of the current study was to investigate alterations in the functional network organization associated with neuroticism. Our findings supported the hypothesis of altered functional connectivity between brain regions in relation to neuroticism, which was based on previous structural connectivity studies that reported widespread decreases in white-matter integrity across multiple fiber 
Table I Results for the Association between Neuroticism and the Network Measures

\begin{tabular}{|c|c|c|c|c|}
\hline & \multicolumn{2}{|c|}{ Binary } & \multicolumn{2}{|c|}{ Weighted } \\
\hline & Threshold values & AUC ( $p$-value) & Threshold values & AUC ( $p$-value) \\
\hline \multicolumn{5}{|l|}{ Whole-brain network } \\
\hline Global efficiency & $0.05-0.30$ & 0.003 & $0.10-0.30$ & 0.017 \\
\hline Local efficiency & $0.13-0.30$ & 0.019 & $0.07-0.30$ & 0.008 \\
\hline \multicolumn{5}{|l|}{ Subnetworks } \\
\hline \multicolumn{5}{|l|}{ Local efficiency } \\
\hline AS & $0.04-0.19$ & 0.017 & - & 0.387 \\
\hline $\cos$ & - & 0.286 & - & 0.175 \\
\hline DMS & - & 0.258 & $0.12-0.30$ & 0.028 \\
\hline \multicolumn{5}{|l|}{ Participation coefficient } \\
\hline AS & $0.07-0.13$ & 0.084 & $0.07-0.13$ & 0.084 \\
\hline $\cos$ & $0.01,0.03-0.30$ & $<0.001$ & $0.01,0.03-0.30$ & $<0.00$ I \\
\hline DMS & - & 0.075 & - & 0.142 \\
\hline FPS & $0.13-0.26,0.29,0.30$ & 0.043 & - & 0.078 \\
\hline SMS & $0.15-0.30$ & 0.023 & $0.19-0.30$ & 0.028 \\
\hline VS & - & 0.363 & - & 0.517 \\
\hline
\end{tabular}

Abbreviations: AS, affective subnetwork; COS, cingulo-operculum subnetwork; DMS, default mode subnetwork; FPS, fronto-parietal subnetwork; SMS, somatosensory-motor subnetwork; VS, visual subnetwork.

The measures global efficiency, local efficiency, maximized modularity, and participation coefficient were calculated on the whole-brain network and/or different functional subnetworks for binary and weighted graphs. In the first column, (the range of) threshold values are listed for which a significant correlation ( $p<0.05$, uncorrected for the number of threshold values) was found between a network measure and neuroticism. These results are indicated with an asterisk (*) in Figures I and 2. In the second column, $p$-values are listed for a one-tailed test of the null hypothesis $(p<0.05)$ wherein we assessed whether the observed area under the curve (AUC) could have occurred by chance.

tracts interconnecting different parts of the brain (Bjørnebekk et al, 2013; Xu and Potenza, 2012). Specifically, we found that the functional network structure of individuals with higher levels of neuroticism is organized less optimally in terms of efficient information processing and consists of weaker functional connections. Furthermore, in high compared with low neurotic individuals, we showed that subnetworks related to emotion and salience processing have a more prominent role in the network organization, while subnetworks related to sensory(-motor) functions and cognitive control have a less prominent role (Doucet et al, 2011; Kinnison et al, 2012; Laird et al, 2011). This is in line with previous studies demonstrating that individuals with higher levels of neuroticism show more difficulties in adaptive emotion regulation (Suls and Martin, 2005; Watson and Hubbard, 1996; Yoon et al, 2013).

\section{Whole-Brain Network Organization in Association with Neuroticism}

In high compared with low neurotic individuals, we observed a network structure that resembles more that of a random network, indicating a loss of optimal small-world characteristics. The weakness of network structures that are organized more randomly is that they are less fault tolerant (less resilient) in case of an adverse perturbation (Latora and Marchiori, 2001) and economically less cost-efficient (see for a discussion, Achard and Bullmore, 2007). Evidence for a more random network structure in individuals with higher levels of neuroticism was demonstrated by two findings. First, we found higher global efficiency and lower local efficiency for binary graphs in association with neuroticism. This has also been found in MDD, for which neuroticism is a risk factor, and other disorders, such as Alzheimer's disease, schizophrenia, and epilepsy (Xia and He, 2011), providing suggestive evidence for the vulnerability of random network structures. Second, in individuals scoring higher on neuroticism, we found that nodes could be delineated less clearly in isolated functional subnetworks, compared with individuals scoring lower (ie, maximized modularity was decreased). Complex systems, such as brain, consist of simplistic subsystems (modules) that are built hierarchically (Simon, 1962). A modular construction is argued to be essential for the optimal performance of such systems and the capability to support an array of diverse behaviors (Simon, 1962). Possibly, when less segregation exists between functional subnetworks, noise is able to propagate and interfere with (task-relevant) information 


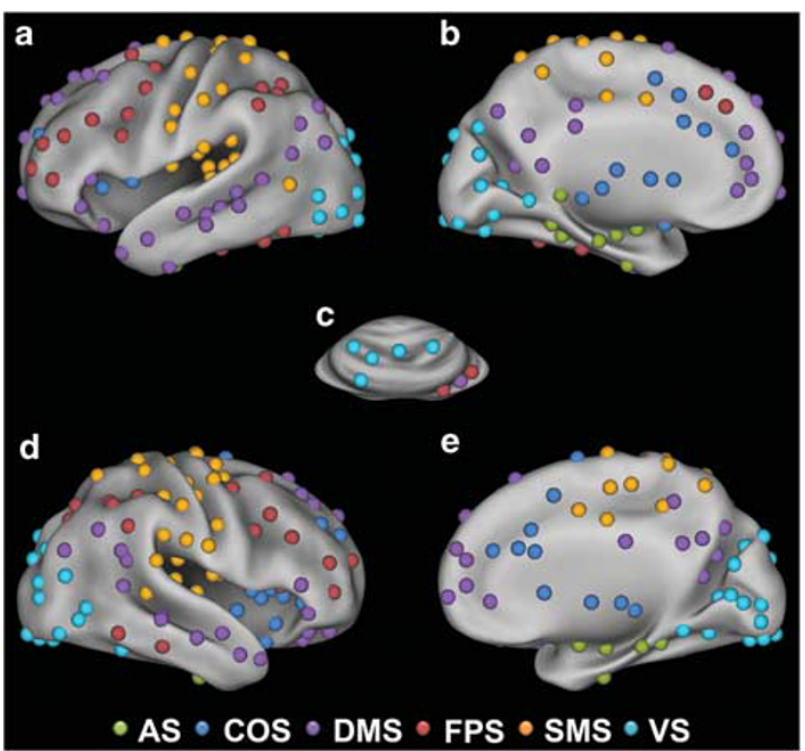

Figure 3 Module decomposition. Nodes could be partitioned in six functional subnetworks with a maximum number of within-group edges and a minimum number of between-group edges. Colors indicate the different modules that nodes belong to: AS, affective subnetwork (green); COS, cingulo-operculum subnetwork (dark blue); DMS, default mode subnetwork (purple); FPS, fronto-parietal subnetwork (red); SMS, somatosensory-motor subnetwork (orange); VS, visual subnetwork (light blue). Nodes are pasted on an inflated surface rendering of the human brain using CARET (v5.65). In the panels, different views are shown: (a) left lateral, (b) left medial, (c) cerebellum dorsal, (d) right lateral, and (e) right medial.

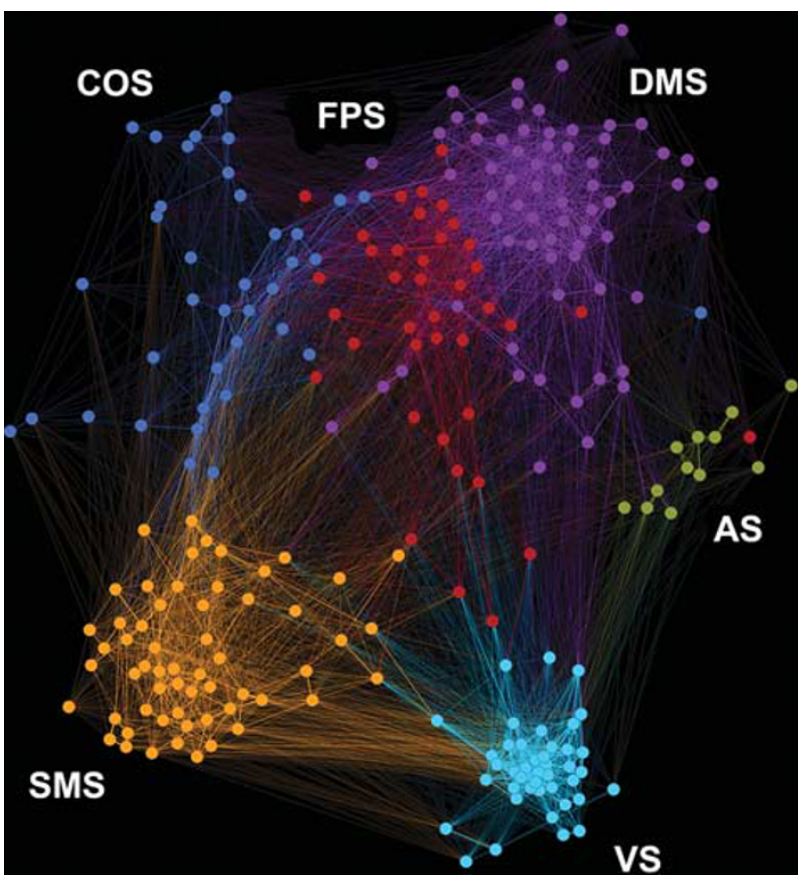

Figure 4 Graph layout. The average graph across subjects is visualized using Gephi (0.8.2-beta, force atlas 2) for the optimal threshold value of $1.8 \%$ (see Supplementary Material S4 for details on the selection of the optimal threshold). Colors indicate the different modules that nodes belong to: AS, affective subnetwork (green); COS, cingulo-operculum subnetwork (dark blue); DMS, default mode subnetwork (purple); FPS, fronto-parietal subnetwork (red); SMS, somatosensory-motor subnetwork (orange); and VS, visual subnetwork (light blue). processing (Stevens et al, 2012) (see Supplementary Material S8 for a dynamic graph that shows how the network structure changes across participants when individuals score higher on neuroticism).

Furthermore, in high compared with low neurotic individuals, we observed a decrease in global and local efficiency for weighted graphs. The reason for this was that individuals scoring higher on neuroticism have overall weaker functional connections between nodes than individuals scoring lower. Having weaker functional connections may disrupt the exchange of information between different brain regions. It is possible that decreases in white-matter integrity, as found in structural connectivity studies on neuroticism (Bjørnebekk et al, 2013; Xu and Potenza, 2012), may explain this observation. In accordance with this suggestion, it has been shown that resting-state functional connectivity is strongly related to the underlying structural connectivity architecture (van den Heuvel et al, 2009) and that the strength of structural connections predicts the strength of functional connections derived from rs-fMRI (Honey et al, 2009). Notably, a combination of decreased functional connectivity and a loss of white-matter integrity has been observed in aging, multiple sclerosis, and schizophrenia (van den Heuvel et al, 2009), suggesting that such disruptions may be a general correlate of suboptimal brain function.

In conclusion, individuals scoring higher on neuroticism have a more random network structure that consists of weaker functional connections, compared with individuals scoring lower. These findings emphasize the importance of calculating network measures on both binary and weighted graphs, since they provide different kinds of information about the functional network organization, ie, binary networks (an edge is present or not) provide information on the functional structure of the network organization and weighted networks (edge strengths are preserved) additionally provide information on the functional connectivity strength.

\section{Organization of the Functional Subnetworks in Association with Neuroticism}

Besides describing the whole-brain properties of the functional network organization in neuroticism, we investigated connectivity within and between functional subnetworks. In high compared with low neurotic individuals, we observed that the AS shows higher local efficiency (in terms of functional structure) and the COS, as well as the AS and the SMS, have relatively more connections with other functional subnetworks. In contrast, we found that sensory(-motor) (SMS and VS) and cognitive control subnetworks (DMS and FPS) process information less efficiently in these high neurotic individuals. Notably, AS and COS consist of brain regions related to the identification and appraisal of salient affective stimuli and the production of affective states (eg, amygdala, hippocampus, and anterior insula) (Kinreich et al, 2011; Laird et al, 2011; Menon and Uddin, 2010; Seeley et al, 2007), while DMS and FPS consist of brain regions involved in attention, memory, emotion regulation, self-reflection, problem solving, and planning (eg, prefrontal and parietal areas) (Buckner et al, 2008; Laird et al, 2011; Seeley et al, 2007; Vincent et al, 2008). Taking these two findings together, this seems to be a recipe 
for emotional instability: subnetworks related to emotion and salience processing have a more prominent role in the functional network organization of individuals scoring higher on neuroticism, while subnetworks that potentially control and regulate responses from these subnetworks have a less prominent role (Doucet et al, 2011; Kinnison et al, 2012; Laird et al, 2011). This is in line with evidence showing that high compared with low neurotic individuals experience more intense negative emotions, such as feelings of depression and anxiety, and demonstrate heighted emotional reactivity to negative events (Suls and Martin, 2005; Watson et al, 1994). Likewise, previous studies have revealed that these high trait scorers deal poorly with (daily) stressors and often apply maladaptive coping strategies, such as worry and avoidance (Suls and Martin, 2005; Watson and Hubbard, 1996; Yoon et al, 2013). These findings may indicate that the 'neurotic brain' is less cognitively controlled and that (negative) affect predominates in the information processing.

\section{Strengths and Limitations}

The current study has two important strengths. First, due to the large sample size and the fact that we selected individuals across the entire range of neuroticism scores, our study was highly sensitive to detect small linear effects in the association between neuroticism and the different network measures. Second, we applied graph theory analyses on the whole brain as well as the different functional subnetworks for both binary and weighted graphs. Furthermore, the current study has also three limitations. First, we did not collect DTI data during our experiment, because of this we cannot verify that a loss of white-matter integrity lies underneath the observed disruptions in the functional network organization of individuals scoring higher on neuroticism. Second, we only investigated female students, because of this our findings cannot be generalized to the population. Future studies should replicate our results in, for example, male samples. However, by selecting a homogenous sample, we controlled for several important confounders, such as gender, age, education level, and ethnicity, which increased our power. Third, we used a univariate approach in the current study. It is known that univariate analyses do not capture the full complexity of brain networks (Simpson et al, 2013). Multivariate approaches are currently being developed to investigate the complex dependence structure of networks and the effects of multiple network measures on the network organization (Simpson et al, 2013). For this reason, our results should be considered exploratory and further validation is needed.

\section{CONCLUSION}

In the current study, we examined alterations in the functional network organization associated with neuroticism. The findings revealed that the topological properties of the 'neurotic brain' showed disrupted functional integration and segregation, suggesting a brain that processes information less cost-efficiently (Achard and Bullmore, 2007). This has also been observed in various other disorders and may be a general mechanism that underlies suboptimal brain function (Bullmore and Sporns, 2012). Furthermore, in high compared with low neurotic individuals, we found that subnetworks related to emotion and salience processing have a more prominent role in the network organization, while subnetworks related to sensory(-motor) functions and cognitive control have a less prominent role. This result may indicate a mechanism that is more specific to neuroticism and may explain why high neurotic individuals are more vulnerable to develop psychopathology, specifically depression and anxiety disorders (Lahey, 2009; Ormel et al, 2013b). Future studies on neuroticism should investigate the association between structural and functional networks, and the association between network measures and behavioral measures of (maladaptive) emotion processing and regulation (Bullmore and Sporns, 2012). On the basis of the acquired knowledge, it may be possible to develop treatments that prevent individuals with higher levels of neuroticism to transit from a healthy state to a clinical state.

\section{FUNDING AND DISCLOSURE}

The authors declare no conflict of interest.

\section{ACKNOWLEDGEMENTS}

The current study was supported by the Ministry of Education, Culture, and Science of the Netherlands (609022). The sponsor did not play a role in the study design; collection, analysis, and interpretation of the data; writing the report or the decision to submit the article for publication. Furthermore, we would like thank A Sibeijn-Kuiper and J Streurman-Werdekker for their support in the data acquisition and $\mathrm{C}$ Levallois for his help in building the dynamic graph in Gephi.

\section{REFERENCES}

Achard S, Bullmore E (2007). Efficiency and cost of economical brain functional networks. PLoS Comput Biol 3: e17.

Andreano JM, Cahill L (2010). Menstrual cycle modulation of medial temporal activity evoked by negative emotion. Neuroimage 53: 1286-1293.

Ashburner J (2007). A fast diffeomorphic image registration algorithm. Neuroimage 38: 95-113.

Bjørnebekk A, Fjell AM, Walhovd KB, Grydeland H, Torgersen S, Westlye LT (2013). Neuronal correlates of the five factor model (FFM) of human personality: multimodal imaging in a large healthy sample. Neuroimage 65: 194-208.

Blondel VD, Guillaume J, Lambiotte R, Lefebvre E (2008). Fast unfolding of communities in large networks. J Stat Mech 10: P10008.

Buckner RL, Andrews-Hanna J, Schacter DL (2008). The brain's default network: anatomy, function, and relevance to disease. In: Kingstone AMiller MB (ed). The Year in Cognitive Neuroscience 2008. Blackwell Publishing: Malden, pp 1-38.

Bullmore E, Sporns O (2012). The economy of brain network organization. Nat Rev Neurosci 13: 336-349.

Cohen BL, Katz M (1979). Pituitary and ovarian function in women receiving hormonal contraception. Contraception 20: 475-487.

Costa PTJ, McCrae RR (1992). Revised NEO Personality Inventory (NEO-PI-R) and the Five Factor Inventory (NEO-FFI): Professional Manual. Psychological Assessment Resources Inc: Odessa, FL.

Cremers HR, Demenescu LR, Aleman A, Renken R, van Tol MJ, van der Wee NJ et al (2010). Neuroticism modulates amygdala- 
prefrontal connectivity in response to negative emotional facial expressions. Neuroimage 49: 963-970.

Damoiseaux JS, Rombouts SA, Barkhof F, Scheltens P, Stam CJ, Smith SM et al (2006). Consistent resting-state networks across healthy subjects. Proc Natl Acad Sci USA 103: 13848-13853.

Doucet G, Naveau M, Petit L, Delcroix N, Zago L, Crivello F et al (2011). Brain activity at rest: a multiscale hierarchical functional organization. J Neurophysiol 105: 2753-2763.

Eysenck HJ (1967). The Biological Basis of Personality. Charles C. Thomas: Spring-field, IL.

Gao Q, Xu Q, Duan X, Liao W, Ding J, Zhang Z et al (2013). Extraversion and neuroticism relate to topological properties of resting-state brain networks. Front Hum Neurosci 7: 257.

Geerligs L, Renken RJ, Saliasi E, Maurits NM, Lorist MM (2014). A brain wide study of age-related changes in functional connectivity. Cereb Cortex (in press).

Goldstein JM, Jerram M, Abbs B, Whitfield-Gabrieli S, Makris N (2010). Sex differences in stress response circuitry activation dependent on female hormonal cycle. J Neurosci 30: 431-438.

Gray JA (1991). Neural systems, emotion and personality. In: Madden JIV (ed). Neurobiology of Learning, Emotion and Affect. Raven Press: New York, USA.

Honey CJ, Sporns O, Cammoun L, Gigandet X, Thiran JP, Meuli R et al (2009). Predicting human resting-state functional connectivity from structural connectivity. Proc Natl Acad Sci USA 106: 2035-2040.

Jin C, Gao C, Chen C, Ma S, Netra R, Wang Y et al (2011). A preliminary study of the dysregulation of the resting networks in first-episode medication-naive adolescent depression. Neurosci Lett 503: 105-109.

Kinnison J, Padmala S, Choi J, Pessoa L (2012). Network analysis reveals increased integration during emotional and motivational processing. J Neurosci 32: 8361-8372.

Kinreich S, Intrator N, Hendler T (2011). Functional cliques in the amygdala and related brain networks driven by fear assessment acquired during movie viewing. Brain Connect 1: 484-495.

Lahey BB (2009). Public health significance of neuroticism. Am Psychol 64: 241-256.

Laird AR, Fox PM, Eickhoff SB, Turner JA, Ray KL, McKay DR et al (2011). Behavioral interpretations of intrinsic connectivity networks. J Cogn Neurosci 23: 4022-4037.

Latora V, Marchiori M (2001). Efficient behavior of small-world networks. Phys Rev Lett 87: 198701.

Menon V, Uddin LQ (2010). Saliency, switching, attention and control: a network model of insula function. Brain Struct Funct 214: $655-667$.

Newman ME (2004). Fast algorithm for detecting community structure in networks. Phys Rev E Stat Nonlin Soft Matter Phys 69: 066133 .

Ormel J, Bastiaansen A, Riese H, Bos EH, Servaas M, Ellenbogen M et al (2013a). The biological and psychological basis of neuroticism: current status and future directions. Neurosci Biobehav Rev 37: 59-72.

Ormel J, Jeronimus BF, Kotov R, Riese H, Bos EH, Hankin B et al (2013b). Neuroticism and common mental disorders: meaning and utility of a complex relationship. Clin Psychol Rev 33: 686-697.

Parker G, Brotchie H (2010). Gender differences in depression. Int Rev Psychiatry 22: 429-436.

Power JD, Barnes KA, Snyder AZ, Schlaggar BL, Petersen SE (2012). Spurious but systematic correlations in functional connectivity MRI networks arise from subject motion. Neuroimage 59: 2142-2154.

Power JD, Cohen AL, Nelson SM, Wig GS, Barnes KA, Church JA et al (2011). Functional network organization of the human brain. Neuron 72: 665-678.
Rubinov M, Sporns O (2010). Complex network measures of brain connectivity: Uses and interpretations. Neuroimage 52: 1059-1069.

Seeley WW, Menon V, Schatzberg AF, Keller J, Glover GH, Kenna $\mathrm{H}$ et al (2007). Dissociable intrinsic connectivity networks for salience processing and executive control. J Neurosci 27: 2349-2356.

Servaas MN, Riese H, Renken RJ, Marsman JB, Lambregs J, Ormel J et al (2013a). The effect of criticism on functional brain connectivity and associations with neuroticism. PLoS ONE 8: e69606.

Servaas MN, van der Velde J, Costafreda SG, Horton P, Ormel J, Riese $\mathrm{H}$ et al (2013b). Neuroticism and the brain: A quantitative meta-analysis of neuroimaging studies investigating emotion processing. Neurosci Biobehav Rev 37: 1518-1529.

Simon HA (1962). The architecture of complexity. Proc Am Philos Soc 106: 467-482.

Simpson SL, Bowman FD, Laurienti PJ (2013). Analyzing complex functional brain networks: fusing statistics and network science to understand the brain. Stat Surv 7: 1-36.

Stevens AA, Tappon SC, Garg A, Fair DA (2012). Functional brain network modularity captures inter- and intra-individual variation in working memory capacity. PLoS One 7: e30468.

Suls J, Martin R (2005). The daily life of the garden-variety neurotic: reactivity, stressor exposure, mood spillover, and maladaptive coping. J Pers 73: 1485-1510.

Sun Y, Danila B, Josic K, Bassler KE (2009). Improved community structure detection using a modified fine-tuning strategy. EPL 86: 28004.

Symonds CS, Gallagher P, Thompson JM, Young AH (2004). Effects of the menstrual cycle on mood, neurocognitive and neuroendocrine function in healthy premenopausal women. Psychol Med 34: 93-102.

van den Heuvel MP, Mandl RCW, Kahn RS, Pol HEH (2009). Functionally linked resting-state networks reflect the underlying structural connectivity architecture of the human brain. Hum Brain Mapp 30: 3127-3141.

Van Dijk KR, Hedden T, Venkataraman A, Evans KC, Lazar SW, Buckner RL (2010). Intrinsic functional connectivity as a tool for human connectomics: theory, properties, and optimization. J Neurophysiol 103: 297-321.

van Wijk BC, Stam CJ, Daffertshofer A (2010). Comparing brain networks of different size and connectivity density using graph theory. PLoS One 5: e13701.

Vincent JL, Kahn I, Snyder AZ, Raichle ME, Buckner RL (2008). Evidence for a frontoparietal control system revealed by intrinsic functional connectivity. J Neurophysiol 100: 3328-3342.

Watson D, Clark LA, Harkness AR (1994). Structures of personality and their relevance to psychopathology. J Abnorm Psychol 103: 18-31.

Watson D, Hubbard B (1996). Adaptational style and dispositional structure: coping in the context of the five-factor model. J Pers 64: 737-774.

Watts DJ, Strogatz SH (1998). Collective dynamics of 'small-world' networks. Nature 393: 440-442.

Xia M, He Y (2011). Magnetic resonance imaging and graph theoretical analysis of complex brain networks in neuropsychiatric disorders. Brain Connect 1: 349-365.

Xu J, Potenza MN (2012). White matter integrity and five-factor personality measures in healthy adults. Neuroimage 59: 800-807.

Yoon KL, Maltby J, Joormann J (2013). A pathway from neuroticism to depression: examining the role of emotion regulation. Anxiety Stress Coping 26: 558-572.

Zhang J, Wang J, Wu Q, Kuang W, Huang X, He Y et al (2011). Disrupted brain connectivity networks in drug-naive, firstepisode major depressive disorder. Biol Psychiatry 70: 334-342.

Supplementary Information accompanies the paper on the Neuropsychopharmacology website (http://www.nature.com/npp) 